\title{
Biological Species of Armillaria in Japan
}

Y. Ota, Forest Pathologist, Forestry and Forest Products Research Institute, Ibaraki, 305, Japan; N. Matsushita, Forest Pathologist, The University of Tokyo; E. Nagasawa, Mycologist, Tottori Mycological Institute, Tottori, 68911, Japan; T. Terashita, Ex-Professor, Kagoshima University; K. Fukuda, Lecturer, and K. Suzuki, Professor, The University of Tokyo, Tokyo 113, Japan

\begin{abstract}
Ota, Y., Matsushita, N., Nagasawa, E., Terashita, T., Fukuda, K., and Suzuki, K. 1998. Biological species of Armillaria in Japan. Plant Dis. 82:537-543.

Pairing tests with isolates of Armillaria in culture were used to identify species of Armillaria and their distribution throughout Japan. The existence of 10 intersterile groups of Armillaria was determined by pairing haploid single spore isolates obtained from 20 basidiocarps from a wide geographic distribution in Japan. Two to four haploid tester isolates from each intersterile group were then paired to biological and morphological species with haploid tester isolates from Europe and North America identified in previous studies. Japanese haploid tester isolates were then paired with 190 haploid or diploid isolates and compatibility reactions were used to identify these to species. Of the 10 intersterile groups identified in Japan, 7 were authenticated as $A$. gallica, A. nabsnona, A. ostoyae, A. cepistipes, A. mellea, A. sinapina, or A. tabescens. Three of the groups were not compatible with any of the tester species. The distribution and host relationships of the Japanese biological species are also discussed.
\end{abstract}

Fungi belonging to the genus Armillaria (Fr.:Fr.) Staude cause root diseases of forest trees, resulting in extensive economic losses to a range of crop plants all over the world (13). In Japan, Armillaria spp. are widely distributed throughout the country and are well-known as a cause of root rots (25).

Armillaria root disease was first reported on Castanea crenata in 1903 (22). Since then, Armillaria root rot has been reported in many cultivated tree species. Damage was particularly severe in pure Larix kaempferi plantations in Hokkaido island, the northern area of Japan, in the 1960s (23). Recently, Armillaria root rot has been reported in plantations of young Hinoki (Chamaecyparis obtusa), which is one of the most important cultivated tree species in Japan (20).

Pairing tests based on sexual behavior and in vitro somatic compatibility of isolates of Armillaria have been widely used to assess interspecific incompatibility in the genus Armillaria. Haploid monosporous cultures exhibit a white, fluffy colony morphology. After compatible mating, the culture morphology becomes crustose, which is characteristic of diploid mycelium. Pairing tests have distinguished biological species in Europe, North Amer-

Corresponding author. Y. Ota

E-mail: yuota@ffpri.affrc.go.jp

Accepted for publication 21 January 1998.

Publication no. D-1998-0309-01R

(C) 1998 The American Phytopathological Society ica, and Australasia $(1,9,15,17)$. Except for the North American species X and XI, all of these biological species either correspond to known morphological species or are described as new species $(3,4,9,15-$ 17,29). In annulate Armillaria spp., 5 species have been established in Europe, 9 in North America, and 5 in Australasia (9). In exannulate Armillaria spp., 2 species have been reported in Europe and 1 in North America (9). All are heterothallic with a bifactorial mating system, with the exception of the European exannulate species $A$. ectypa (9).

In Japan, Terashita and Chuman (27) recognized 5 biological species from Galeola septentrionalis, an achlorophyllos orchid. A. borealis, A. gallica (as A. bulbosa), A. cepistipes, A. mellea, and A. tabescens were isolated from Kyushu, and 2 biological species, A. gallica and $A$. cepistipes, were isolated from Honshu. Nagasawa (21) recorded 6 intersterility groups (designated as A, B, C, D, E, and AM) in Honshu. Based on the morphological characters of their basidiocarps, he considered that groups $\mathrm{C}$ and $\mathrm{AM}$ were identical with A. ostoyae and A. mellea, respectively; groups $\mathrm{A}$ and $\mathrm{D}$ were similar to A. gallica (as A. lutea) and A. cepistipes, respectively; and $\mathrm{B}$ and $\mathrm{E}$ represented new species. The 6 intersterility groups were recognized as distinct by Matsushita et al. (18) using isozyme analysis. From pairing tests with European and North American biological species, Suzuki (25) and Suzuki et al. (26) have suggested that Nagasawa's (Nag.) A, Nag. B, Nag. C, Nag. D, and Nag. AM are equivalent to A. gallica, NABS IX (= A. nabsnona), A. ostyae, A. cepistipes, and A. mellea, respectively. The occurrence of biological species A. gallica and A. mellea in Honshu and Kyushu was recently recognized by Mohammad et al. (19). Also, 8 biological species, A. gallica (as A. bulbosa), A. cepistipes, A. ostoyae, A. sinapina, NABS IX, A. mellea subsp. nipponica, A. singula, and A. jezoensis, have been reported from Hokkaido, of which the latter three are new taxa (6$8,19)$.

These previous studies of biological species in Japan were conducted using some isolates from a limited area of Japan. The objectives of this study were to expand the survey for biological species of Armillaria in Japan by obtaining isolates from localities throughout Japan and identifying them to species, and to discuss their distribution and host ranges.

\section{MATERIALS AND METHODS}

Haploid isolates obtained from 20 basidiocarps were used for pairing tests to determine the intersterile groups in Japan. These isolates of Nag. A, B, C, D, E, and AM were obtained from the Tottori Mycological Institute Culture Collection (TMIC) and corresponded to Nagasawa's 6 intersterile groups A, B, C, D, E, and AM (21), respectively. Other monosporous isolates were prepared from single basidiocarps of additional collections. Details of 20 basidiocarps are shown in Table 1 . The mating types of monosporous cultures from each of these 20 basidiocarps were determined by pairing 12 isolates from each basidiocarp in all combinations. The two or four mating types from each basidiocarp were paired with those of the other basidiocarps in all combinations, and the number of intersterile groups was determined. Tester strains from each intersterile group were then selected for pairing with European and North American tester strains from known biological or taxonomic Armillaria spp.

Haploid tester strains from known species of Armillaria in Europe and North America, their hosts, and geographic source are indicated in Tables 2 and 3, respectively. Two to four tester strains were used from each biological species. Japanese testers were paired with them in all combinations. Individual pairings were performed at least three times. The other 190 isolates of Armillaria spp., which were collected in Japan from single spore, stipe 
Table 1. Sources of the Japanese tester isolates of Armillaria spp.

\begin{tabular}{|c|c|c|c|c|c|}
\hline Biological species & Stock number & Nuclear phase $^{a}$ & Host & Location & Original stock number \\
\hline Nag. A & NA 4 & $\mathrm{H}$ & Soil surface & Tateiwa-mura, Fukushima & TMIC 31063 \\
\hline Nag. A & NA 10 & $\mathrm{H}$ & Soil surface & Tottori-shi, Tottori & TMIC 30984 \\
\hline Nag. A & NA 13 & $\mathrm{H}$ & Soil surface & Aomori-shi, Aomori & TMIC 31525 \\
\hline Nag. B & NB 3 & $\mathrm{H}$ & Soil surface & Tottori-shi, Tottori & TMIC 30987 \\
\hline Nag. C & $\mathrm{NC} 2$ & $\mathrm{H}$ & Unknown & Hirama-machi, Aomori & TMIC 30966 \\
\hline Nag. C & NC 3 & $\mathrm{H}$ & Quercus crispula & Appi, Iwate & TMIC 30969 \\
\hline Nag. C & NC 8 & $\mathrm{H}$ & Soil surface & Hiranai-cho, Aomori & TMIC 31491 \\
\hline Nag. D & ND 1 & $\mathrm{H}$ & Fagus crena ta & Towadako-machi, Aomori & TMIC 30968 \\
\hline Nag. D & ND 3 & $\mathrm{H}$ & Betula sp. & Appi, lwate & TMIC 30970 \\
\hline Nag. D & ND 11 & $\mathrm{H}$ & Quercus crispula & Mt. Hakkoda, Aomori & TMIC 31069 \\
\hline Nag. E & NE 1 & $\mathrm{H}$ & Decayed wood & Mt. Hakkoda, Aomori & TMIC 31061 \\
\hline Nag. E & NE 7 & $\mathrm{H}$ & Unknown & Kawahara-cho, Tottori & TMIC 31510 \\
\hline Nag. E & NE 9 & $\mathrm{H}$ & Castanea crenata & Tottori-shi, Tottori & TMIC 31515 \\
\hline Nag. AM & NAM 5 & $\mathrm{U}$ & Hardwood & Tottori-shi, Tottori & TMIC 32415 \\
\hline Nag. AM & NAM 6 & $\mathrm{U}$ & Pyrus pyrifolia & Aoya-cho, Tottori & TMIC 32417 \\
\hline Nag. AM & NAM 7 & $\mathrm{D}$ & Quercus serrata & Kokufu-cho, Tottori & TMIC 32416 \\
\hline $\mathrm{F}$ & $96-7$ & $\mathrm{H}$ & Lalix kaempferi & Chitose-shi, Hokkaido & \\
\hline G & $\mathrm{H}-5$ & $\mathrm{H}$ & Unknown & Kamishihoro-cho, Hokkaido & \\
\hline $\mathrm{H}$ & $\mathrm{H}-16$ & $\mathrm{H}$ & Unknown & Asahikawa-shi, Hokkaido & \\
\hline $\mathrm{T}$ & NT 1 & $\mathrm{H}$ & Quercus myrsinaefolia & Tsukuba-shi, Ibaraki & \\
\hline
\end{tabular}

${ }^{\mathrm{a}} \mathrm{D}=$ diploid (isolated from stipe tissue or mycelial mat ); $\mathrm{H}=$ haploid (isolated from single spore ); $\mathrm{U}=$ single spore culture appear crustose.

Table 2. Species, host, geographic location, and source of European haploid testers of Armillaria spp.

\begin{tabular}{|c|c|c|c|c|c|}
\hline Species & Isolate number & Substrate / host & Geographic location & Original isolate number & source \\
\hline \multirow[t]{2}{*}{ A. borealis } & G-1 & Betula sp. & Ruovesi, Finland & KA1 & K. Korhonen \\
\hline & G-2 & Betula sp. & Hartola, Finland & KA2 & K. Korhonen \\
\hline \multirow[t]{3}{*}{ A. cepistipes } & G-3 & Alnus sp. & Tampere, Finland & KB2 & K. Korhonen \\
\hline & G-4 & Acer pseudoplatanus & Helsinki, Finland & KB3 & K. Korhonen \\
\hline & G-5 & Tilia platyphyllos & Puy-de-Dôme, France & $\mathrm{T} 21$ & J. J. Guillaumin \\
\hline \multirow[t]{3}{*}{ A. ostoyae } & G-6 & Pinus sylvestris & Nurmijärvi, Finland & $\mathrm{KC} 2$ & K. Korhonen \\
\hline & G-7 & Pinus sylvestris & Nurmijärvi, Finland & $\mathrm{KC} 4$ & K. Korhonen \\
\hline & G-8 & Abies pectinata & Puy-de-Dôme, France & SF2.5 & J. J. Guillaumin \\
\hline \multirow[t]{3}{*}{ A. mellea } & G-9 & Unknown & Paris region, France & KDl & K. Korhonen \\
\hline & G-10 & Soil surface & Eure, France & KD4 & K. Korhonen \\
\hline & G-11 & Prunus persica & Pyr6n6es-Orientales, France & PM8 & J. J. Guillaumin \\
\hline \multirow[t]{3}{*}{ A. gallica } & G-12 & Unknown & Near Paris, France & KE1 & K. Korhonen \\
\hline & G-13 & Rubus fruticosus & Puy-de-Dôme, France & $\mathrm{R} 2$ & J. J. Guillaumin \\
\hline & G-14 & Corylus avellana & Puy-de-Dôme, France & ME 80-15-1 & J. J. Guillaumin \\
\hline \multirow[t]{3}{*}{ A. tabescens } & G-15 & Unknown & Puy-de-Dôme, France & MT $80-42-3$ & J. J. Guillaumin \\
\hline & G-16 & Quercus robur & Randan Puy-de-Dôme, France & MT 83-10-8 & J. J. Guillaumin \\
\hline & G-17 & Unknown & Puy-de-Dôme, France & MT 67-6-5 & J. J. Guillaumin \\
\hline
\end{tabular}

Table 3. Species, host, geographic locations, and source of North American haploid testers of Armillaria spp.

\begin{tabular}{|c|c|c|c|c|c|}
\hline Species & Isolate number & Substrate / host & Geographic location & Original isolate number & Source \\
\hline \multirow{3}{*}{ A. ostoyae } & A-3 & Abies balsamea & Mt. Mansfield, VT & $28-4$ & J. B. Anderson \\
\hline & A-7 & Soil surface & Orleans Co., VT & $70-1$ & J. B. Anderson \\
\hline & A-21 & Hardwood sap. & Upper Peninsula, MI & $430-5$ & J. B. Anderson \\
\hline \multirow[t]{2}{*}{ A. gemina } & A-4 & Unknown & Camel's Hump, VT & $35-5$ & J. B. Anderson \\
\hline & A-14 & Unknown & Smuggler's Notch, VT & $160-8$ & J. B. Anderson \\
\hline \multirow[t]{2}{*}{ A. calvescens } & A-1 & Acer saccharum & Underhill, VT & $11-1$ & J. B. Anderson \\
\hline & A-2 & Acer saccharum & Underhill, VT & $21-2$ & J. B. Anderson \\
\hline \multirow{2}{*}{ A. sinapina } & A-5 & Acer sp. & Ithaca, NY & $48-6$ & J. B. Anderson \\
\hline & A-16 & Hardwood stump & Babine Lake, B.C. & Sp 81-1-3 & D. Morrison \\
\hline \multirow[t]{2}{*}{ A. mellea } & A-6 & Unknown & Boston area, MA & $49-8$ & J. B. Anderson \\
\hline & A-9 & Acer rubrum & Provincetown, MA & $97-1$ & J. B. Anderson \\
\hline \multirow[t]{4}{*}{ A. gallica } & A-8 & Fraxinus sp. & Burlington, VT & $90-10$ & J. B. Anderson \\
\hline & A-11 & Unknown & Ann Arbor, MI & $137-1$ & J. B. Anderson \\
\hline & A-20 & Hardwood sap. & Upper Peninsula, MI & $427-4$ & J. B. Anderson \\
\hline & A-23 & Hardwood sap. & Upper Peninsula, MI & $454-2$ & J. B. Anderson \\
\hline \multirow[t]{2}{*}{ A. nabsnona } & A-10 & Acer macrophyllum & Vancouver, B.C. & $121-1$ & J. B. Anderson \\
\hline & A-12 & Soil surface & Moscow, ID & $139-1$ & J. B. Anderson \\
\hline \multirow[t]{2}{*}{ NABS $X$} & A-13 & Abies grandis & Moscow, ID & $140-6$ & J. B. Anderson \\
\hline & A-17 & Conifer & Southeastern B.C. & Sp 81-20-1 & D. Morrison \\
\hline \multirow[t]{2}{*}{ NABS XI } & A-18 & & & Sp $82-14-8$ & D. Morrison \\
\hline & A-19 & & & Sp 82-14-9 & D. Morrison \\
\hline A. tabescens & A-15 & & & $202-1$ & J. B. Anderson \\
\hline
\end{tabular}


tissue, rhizomorphs, decayed wood, and infected roots, were identified by pairing with the Japanese tester strains. Pairings were made on $1.5 \%$ malt extract agar or potato dextrose agar medium, and compatibility was determined on the basis of the macroscopic mycelial appearance (17). Pairing between two compatible cotton haploid mycelium changes the morphology of haploid mycelia into the crustose type. When a cottony haploid mycelium is paired with a crustose diploid mycelium belonging to the same species, it usually changes to the crustose mycelial type (17).

For observation of the presence or absence of clamp connection and basidia, and the number of nuclei, gills were fixed with ethanol:acetic acid solution $(3: 1, \mathrm{vol} / \mathrm{vol})$ and were stained with $500 \mathrm{ng} / \mathrm{ml} \mathrm{4}$,6-dia-

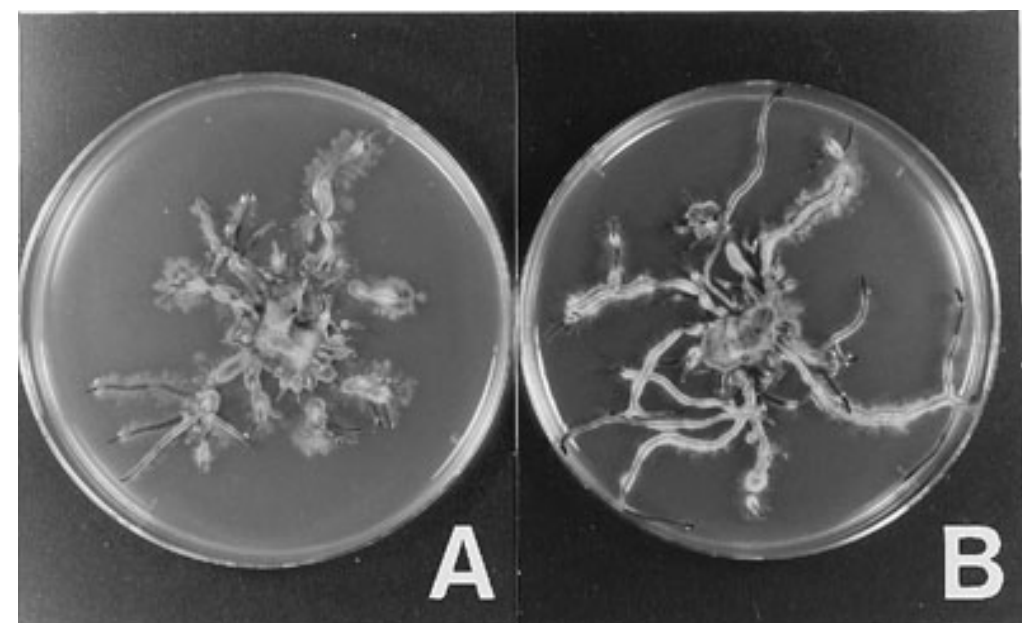

Fig. 1. The culture morphology of Nag. AM (Japanese Armillaria mellea) after 4 weeks' inoculation at $25^{\circ} \mathrm{C}$ on potato dextrose agar. (A) Culture from stipe tissue; (B) culture from a single spore.

Table 4. Results of pairing tests between Japanese and European Armillaria isolates

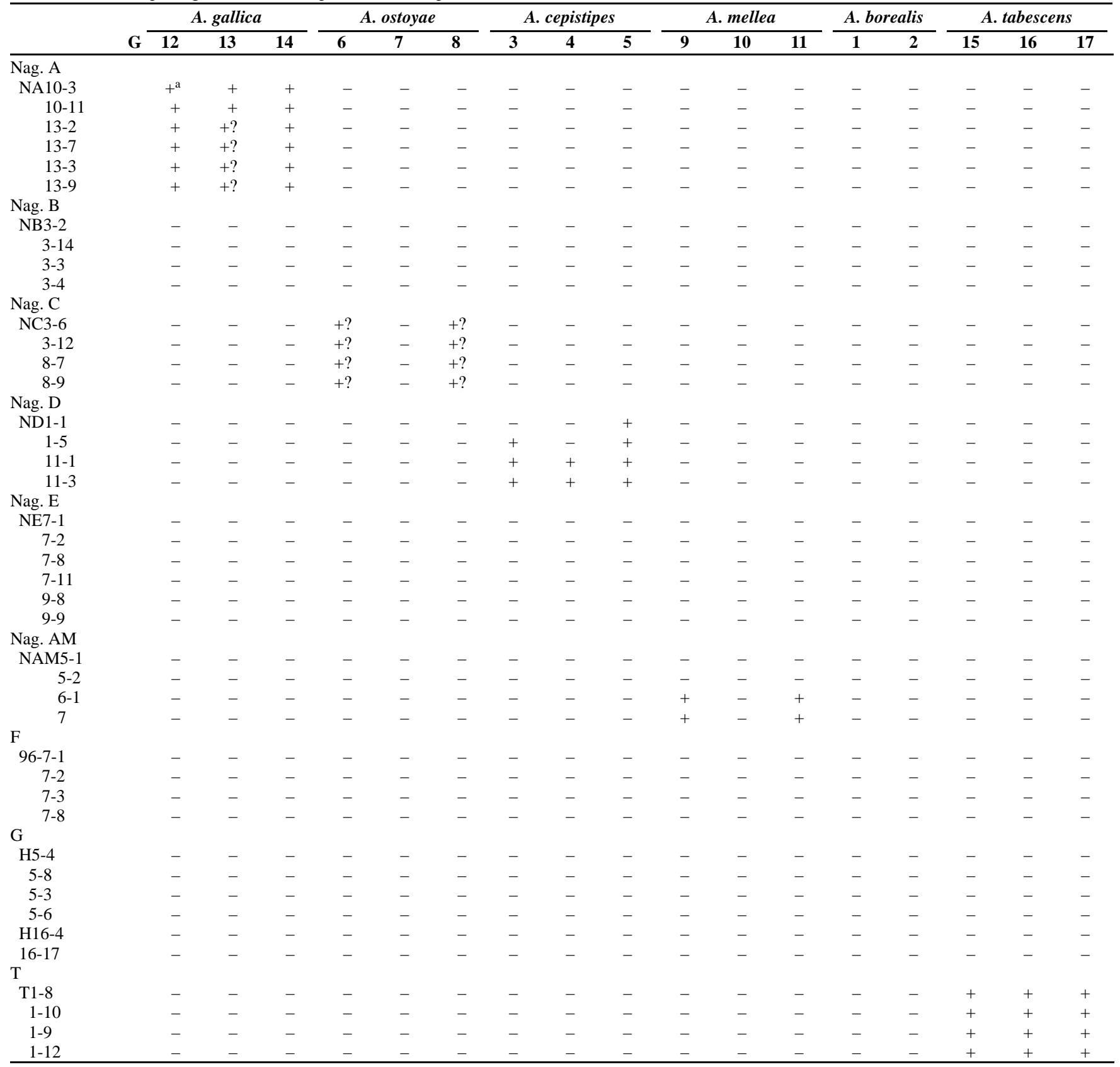

${ }^{\mathrm{a}}+=$ pairing clearly compatible; + ? = pairing not clear but interpreted as compatible; $-=$ = pairing clearly incompatible. 
midino-phenylindol (DAPI) and observed under light and fluorescence microscopes. A. mellea and A. ectypa are known to have clampless uninucleate basidia $(9,21)$. Nagasawa (21) reported that group AM also had clampless basidia. These cytological characteristics can help to identify species.

All isolates used in this study are deposited in the Forestry and Forest Products Institute and the Japanese testers of Nag. A, B, C, D, E, and AM are also deposited in the TMIC.

\section{RESULTS}

Pattern of sexuality. The pattern of sexuality for almost all isolates was clearly bifactorial. Those isolates which did not exhibit bifactorial sexuality possessed a crustose monosporous culture morphology typical of diploid isolates (Fig. 1) and had clampless uninucleate basidia and a similar basidiocarp morphology. Consequently, they were considered to belong to a single group. From this group, 1 or 2 monosporous isolates were randomly selected as tester strains from each basidiocarp. Their compatibility was similarly determined by haploid-diploid pairings.

Grouping of Japanese isolates. From the results of pairing tests among the monosporous isolates from the 20 basidiocarps, the isolates were grouped into 10 biological species: 9 annulate sepcies (Nag. A, Nag. B, Nag. C, Nag. D, Nag. E,
Nag. AM, F, G, and H) and 1 exannulate species (T).

Identification of Japanese groups by haploid-haploid pairing tests with known European and North American testers. The results of pairing tests with European testers and North American testers are shown in Tables 4 and 5, respectively. Three testers (A-6, A-16, and G-7) were considered to be debilitated, because they did not show any morphological change when paired with another tester belonging to same species. In annulate groups, Nag. A was compatible with both European and North American A. gallica. Nag. B was compatible with the North American species A. nabsnona. Nag. C

Table 5. Results of pairing tests between Japanese and North American Armillaria isolates

\begin{tabular}{|c|c|c|c|c|c|c|c|c|c|c|c|c|c|c|c|c|c|c|c|c|c|c|}
\hline \multirow[b]{2}{*}{ A- } & \multicolumn{4}{|c|}{$\begin{array}{c}\text { A. } \\
\text { gallica }\end{array}$} & \multicolumn{2}{|c|}{$\begin{array}{c}A . \\
\text { nabsnona } \\
\end{array}$} & \multicolumn{3}{|c|}{$\begin{array}{c}\text { A. } \\
\text { ostoyae }\end{array}$} & \multicolumn{2}{|c|}{$\begin{array}{c}\text { A. } \\
\text { mellea } \\
\end{array}$} & \multicolumn{2}{|c|}{$\begin{array}{c}A . \\
\text { gemina } \\
\end{array}$} & \multicolumn{2}{|c|}{$\begin{array}{c}\text { A. } \\
\text { calvaescens } \\
\end{array}$} & \multicolumn{2}{|c|}{$\begin{array}{c}A . \\
\text { sinapina } \\
\end{array}$} & \multicolumn{2}{|c|}{ NABSX } & \multicolumn{2}{|c|}{ NABSXI } & \multirow{2}{*}{$\begin{array}{c}A . \\
\text { tabescens } \\
15\end{array}$} \\
\hline & 8 & 11 & 20 & 23 & 10 & 12 & 3 & 7 & 21 & 6 & 9 & 4 & 14 & 1 & 2 & 5 & 16 & 13 & 17 & 18 & 19 & \\
\hline \multicolumn{23}{|l|}{$\overline{\text { Nag. A }}$} \\
\hline NA13-12 & $+?^{\mathrm{a}}$ & + & + & + & - & - & - & - & - & - & - & - & - & - & - & - & - & - & - & - & - & - \\
\hline $13-7$ & $+?$ & + & + & + & - & - & - & - & - & - & - & - & - & - & - & - & - & - & - & - & - & - \\
\hline $13-3$ & $+?$ & + & + & + & - & - & - & - & - & - & - & - & - & - & - & - & - & - & - & - & - & - \\
\hline $13-9$ & $+?$ & + & + & + & - & - & - & - & - & - & - & - & - & - & - & - & - & - & - & - & - & - \\
\hline Nag. B & & & & & & & & & & & & & & & & & & & & & & \\
\hline NB3-2 & - & - & - & - & + & + & - & - & - & - & - & - & - & - & - & - & - & - & - & - & - & - \\
\hline $3-14$ & - & - & - & - & + & + & - & - & - & - & - & - & - & - & - & - & - & - & - & - & - & - \\
\hline $3-3$ & - & - & - & - & + & + & - & - & - & - & - & - & - & - & - & - & - & - & - & - & - & - \\
\hline $3-4$ & - & - & - & - & + & + & - & - & - & - & - & - & - & - & - & - & - & - & - & - & - & - \\
\hline Nag. C & & & & & & & & & & & & & & & & & & & & & & \\
\hline NC3-6 & - & - & - & - & - & - & + & $?$ & $?$ & - & - & - & - & - & - & - & - & - & - & - & - & - \\
\hline $3-12$ & - & - & - & - & - & - & + & $?$ & $?$ & _- & - & _- & - & - & - & _- & - & - & - & - & - & - \\
\hline $8-7$ & - & - & - & - & - & - & + & + ? & + ? & - & - & - & - & - & - & - & - & - & - & - & - & - \\
\hline $8-9$ & - & - & - & - & - & - & + & + ? & $+?$ & - & - & - & - & - & - & - & - & - & - & - & - & - \\
\hline Nag. D & & & & & & & & & & & & & & & & & & & & & & \\
\hline ND11-1 & - & - & - & - & - & $\ldots$ & - & $\ldots$ & $\ldots$ & - & - & - & $\ldots$ & $\ldots$ & - & - & $\ldots$ & $\ldots$ & - & - & - & - \\
\hline $11-3$ & - & - & - & - & - & $\ldots$ & - & $\ldots$ & $\ldots$ & - & - & - & $\ldots$ & $\ldots$ & - & - & $\ldots$ & $\ldots$ & - & - & - & - \\
\hline $13-1$ & - & - & - & - & - & $\ldots$ & - & $\ldots$ & $\ldots$ & - & - & - & $\ldots$ & $\ldots$ & - & - & $\ldots$ & $\ldots$ & - & - & - & - \\
\hline $13-4$ & - & - & - & - & - & $\ldots$ & - & $\ldots$ & $\ldots$ & - & - & _- & $\ldots$ & $\ldots$ & - & - & $\ldots$ & $\ldots$ & - & _- & - & _- \\
\hline Nag. E & & & & & & & & & & & & & & & & & & & & & & \\
\hline NE7-1 & - & - & - & - & - & - & - & - & - & - & - & - & - & - & - & - & - & - & - & - & - & - \\
\hline $7-2$ & - & - & - & - & - & - & - & - & - & - & - & - & - & - & - & - & - & - & - & - & - & - \\
\hline $7-8$ & - & - & - & - & - & - & - & - & - & - & - & - & - & - & - & - & - & - & - & - & - & - \\
\hline $7-11$ & - & - & - & - & - & - & - & - & - & - & - & - & - & - & - & - & - & - & - & - & - & _- \\
\hline $9-8$ & - & - & - & - & - & - & - & - & - & - & - & - & - & - & - & - & - & - & - & - & - & - \\
\hline $9-9$ & - & - & - & - & - & - & - & - & - & - & - & - & - & - & - & - & - & - & - & - & - & - \\
\hline Nag. AM & & & & & & & & & & & & & & & & & & & & & & \\
\hline NAM5-1 & - & - & - & - & - & - & - & - & - & - & - & - & - & - & - & - & - & - & - & - & - & - \\
\hline $5-2$ & - & - & - & - & - & - & - & - & - & - & - & - & - & - & - & - & - & - & - & - & - & - \\
\hline $6-1$ & - & - & - & - & - & - & - & - & - & - & $+?$ & - & - & - & - & - & - & - & - & - & - & - \\
\hline 7 & - & - & - & - & - & - & - & - & - & - & + & - & - & - & - & - & - & - & - & - & - & - \\
\hline $\mathrm{F}$ & & & & & & & & & & & & & & & & & & & & & & \\
\hline $96-7-1$ & - & - & - & - & - & - & - & _- & - & _- & - & _- & - & - & - & + & - & - & - & - & - & - \\
\hline $7-2$ & - & - & - & - & - & - & - & - & - & - & - & - & - & - & - & + & - & - & - & - & - & - \\
\hline $7-3$ & - & - & - & - & - & - & - & - & - & - & - & - & - & - & - & + & - & - & - & - & - & - \\
\hline $7-8$ & - & - & - & - & - & - & - & - & - & - & - & - & - & - & - & + & - & - & - & - & - & - \\
\hline G & & & & & & & & & & & & & & & & & & & & & & \\
\hline H5-4 & - & - & _- & - & - & - & - & _- & - & _- & - & - & - & - & - & - & - & - & - & - & - & - \\
\hline-8 & - & - & - & - & - & - & - & - & - & - & - & - & - & - & - & - & - & - & - & - & - & - \\
\hline-3 & - & - & - & - & - & - & - & - & _- & - & - & - & - & - & - & - & - & - & - & _ & - & - \\
\hline-6 & - & - & - & - & - & - & - & - & - & - & - & - & - & - & - & - & - & - & - & - & - & - \\
\hline $\mathrm{H}$ & & & & & & & & & & & & & & & & & & & & & & \\
\hline H16-4 & - & - & - & - & - & - & - & - & - & - & - & - & - & - & - & - & - & - & - & - & - & - \\
\hline-17 & - & - & - & - & - & - & - & - & - & - & - & - & - & - & - & - & - & - & - & - & - & - \\
\hline $\mathrm{T}$ & & & & & & & & & & & & & & & & & & & & & & \\
\hline NT1-8 & - & - & - & - & - & - & - & - & - & - & - & - & - & - & - & - & - & - & - & - & - & - \\
\hline $1-10$ & - & - & - & - & - & - & - & - & - & - & - & - & - & - & - & - & - & - & - & - & - & - \\
\hline $1-9$ & - & - & - & - & - & - & - & - & - & - & - & - & - & - & - & - & - & - & - & - & - & - \\
\hline $1-12$ & - & - & - & - & - & - & - & - & - & - & - & - & - & - & - & - & - & - & - & - & - & - \\
\hline
\end{tabular}

a $+=$ pairing clearly compatible + ? = pairing not clear but interpreted as compatible $;-=$ pairing clearly incompatible; $-?=$ pairing not clear but interpreted as incompatible; ? = pairing too unclear to give an interpretation; $\ldots=$ no pairing made. 
was compatible with both European and North American A. ostoyae. Nag. D was compatible with the European species $A$. cepistipes. Nag. AM was partially compatible with European and North American A. mellea; NAM6 and NAM7 were compatible with European and North American testers but NAM5 was incompatible with both. Nag. E, group G, and group $\mathrm{H}$ were incompatible with all European and North American testers. Group F was compatible with the North American species $A$. sinapina. In the exannulate group, group $\mathrm{T}$ was compatible with the European A. tabescens but not with North American testers of the same species.

From the results of the pairing tests with Japanese testers, all isolates, with the exception of isolates belonging to Nag. AM, were identified. The isolates belonging to Nag. AM were identified from basidiocarp morphology, monosporous culture colony morphology, and the presence of clampless uninucleate basidia.

Distribution and hosts of Japanese Armillaria spp. The distribution of the isolates of each biological species is shown in Figure 2. A. gallica (Nag. A) was found throughout Japan. A. nabsnona (Nag. B) and Nag. E were found only on Honshu island. A. ostoyae (Nag. C) was found in Hokkaido, and the northern and central parts of Honshu island. A. cepistipes (Nag.
D) was found in Hokkaido and throughout Honshu island. A. sinapina $(\mathrm{F})$, A. singula, $(\mathrm{G})$ and $A$. jezoensis $(\mathrm{H})$ were found only in Hokkaido. A. tabescens ( $\mathrm{T}$ ) was found in Kyushu and the central and southern parts of Honshu.

The number of collections of each $A r$ millaria species on each host species or substrate and their conditions are shown in Tables 6 and 7. A. gallica, A. nabsnona, A. cepistipes, A. tabescens, and Nag. E were found more frequently on hardwoods than on conifers. A. ostoyae and A. mellea were found on both hardwoods and conifers. $A$. mellea was strongly parasitic on Chamae- cyparis obtusa. All C. obtusa hosts, from which 4 isolates of A. mellea were isolated, were either infected or killed (Table 7).

\section{DISCUSSION}

Japan consists of a group of islands extending north and south over a wide range of latitude. As a result, the climate ranges from cold-temperate in the north to subtropical in the south. Consequently, a great diversity of vegetational and mycological flora exists within Japan. From this study, it is clear that 9 annulate species and 1 exannulate species of Armillaria exist in Japan. This number should be recognized

Table 7. Number of collections on tree conditions of Armillaria species identified in Japan

\begin{tabular}{|c|c|c|c|c|c|c|c|c|}
\hline \multirow[b]{2}{*}{ Species } & \multicolumn{3}{|c|}{ Conifers } & \multicolumn{3}{|c|}{ Hardwoods } & \multirow[b]{2}{*}{ Others } & \multirow[b]{2}{*}{ Total $^{b}$} \\
\hline & $\mathbf{P a}$ & $\mathbf{S}$ & $\mathbf{U}$ & $\mathbf{P}$ & $\mathbf{S}$ & $\mathbf{U}$ & & \\
\hline$\overline{\text { A. gallica }}$ & 0 & 1 & 1 & 0 & 6 & 2 & 31 & 41 \\
\hline A. nabsnona & 0 & 0 & 0 & 0 & 2 & 0 & 7 & 9 \\
\hline A. ostoyae & 2 & 6 & 2 & 0 & 7 & 2 & 10 & 29 \\
\hline A. cepistipes & 0 & 0 & 1 & 0 & 14 & 3 & 11 & 29 \\
\hline Nag. E & 0 & 1 & 0 & 0 & 16 & 11 & 8 & 36 \\
\hline A. mellea & 4 & 1 & 0 & 4 & 18 & 2 & 5 & 34 \\
\hline A. sinapina & 0 & 0 & 0 & 0 & 0 & 1 & 2 & 3 \\
\hline $\mathrm{G}$ & 0 & 0 & 0 & 0 & 0 & 0 & 5 & 5 \\
\hline $\mathrm{H}$ & 0 & 0 & 0 & 0 & 0 & 0 & 15 & 15 \\
\hline A. tabescens & 0 & 0 & 0 & 0 & 1 & 3 & 5 & 9 \\
\hline
\end{tabular}

${ }^{a} \mathrm{P}=$ parasite (infection of the cambium of a live tree, mycelial fan penetrating into live cambium of root collar or roots); $\mathrm{S}=$ saprophyte (sap rot of a stump or log); $\mathrm{U}=$ unknown.

${ }^{\mathrm{b}}$ Total $=210$ isolates.

Table 6. Number of collections on each host or substrate of Armillaria spp. identified in Japan

\begin{tabular}{|c|c|c|c|c|c|c|c|c|c|c|}
\hline & A. gallica & A. nabsnona & A. ostoyae & A. cepistipes & Nag. E & A. mellea & A. sinapina & $\mathbf{G}$ & $\mathbf{H}$ & A. tabescens \\
\hline \multicolumn{11}{|l|}{ Conifers } \\
\hline Abies sachalinensis & 1 & & 4 & & & & & & & \\
\hline Abies spp. & & & 2 & & & & & & & \\
\hline \multicolumn{11}{|l|}{ Chamaecyparis } \\
\hline obtusa & & & & & & 4 & & & & \\
\hline Larix kaempferi & & & 1 & 1 & & & & & & \\
\hline \multicolumn{11}{|l|}{ Metasequoia } \\
\hline glyptostroboides & & & & & 1 & & & & & \\
\hline Picea jezoensis & & & 2 & & & & & & & \\
\hline Pinus densiflora & 1 & & 1 & & & & & & & \\
\hline Pinus thunbergii & & & & & & 1 & & & & \\
\hline \multicolumn{11}{|l|}{ Hardwoods } \\
\hline Acer spp. & 1 & & 1 & & 1 & 1 & & & & \\
\hline Aphananthe aspera & & & & & 1 & & & & & \\
\hline Aesculus turbinata & & & & & & 1 & & & & \\
\hline \multicolumn{11}{|l|}{ Betula } \\
\hline maximowicziana & 1 & & 2 & & & & & & & \\
\hline Betula spp. & & & 3 & 1 & & & & & & \\
\hline Castanea crenata & & & & & 1 & 1 & & & & \\
\hline \multicolumn{11}{|l|}{ Cercidiphyllum } \\
\hline japonicum & & & & 1 & 1 & 1 & & & & \\
\hline Fagus crenata & & & & 8 & 5 & 1 & & & & \\
\hline Prunus spp. & & & & & & 3 & & & & 2 \\
\hline Pyrus spp. & & & & & & 1 & & & & \\
\hline Quercus crispula & & & 1 & 1 & 1 & & & & & \\
\hline Quercus serrata & 2 & & & & & 2 & & & & \\
\hline Quercus spp. & 1 & 1 & 2 & 1 & 1 & 1 & & & & 2 \\
\hline Other hardwoods & 3 & 1 & & 5 & 16 & 12 & 1 & & & \\
\hline \multicolumn{11}{|l|}{ Others } \\
\hline Moss & & 1 & & & & & & & & \\
\hline Soil surface & 18 & 4 & 7 & 1 & & & & & & \\
\hline $\begin{array}{l}\text { Galeola } \\
\text { septentrionali }\end{array}$ & 9 & & & 4 & & 2 & & & & 5 \\
\hline Unidentified hosts & 4 & 2 & 3 & 6 & 8 & 3 & 2 & 5 & 15 & \\
\hline Total $^{\mathrm{a}}$ & 41 & 9 & 29 & 29 & 36 & 34 & 3 & 5 & 15 & 9 \\
\hline
\end{tabular}

${ }^{\text {a }}$ Total $=210$ isolates. 
as a lower limit, because the subtropical regions of Japan (Nansei islands, Ogasawara islands, and so on) were not surveyed. Seven groups of Armillaria in Japan were identified by pairing tests with European and North American species. A. gallica (= Nag. A), A. nabsnona (= Nag. B), A. ostoyae (= Nag. C), A. cepistipes (= Nag. D), A. mellea (= Nag. AM), A. sinapina $(=\mathrm{F})$, and $A$. tabescens $(=\mathrm{T})$ were confirmed to exist in Japan. $A$. nabsnona, which has been reported in Hokkaido (19), was first recorded in Honshu. Terashita and Chuman (27) reported the European biological species, A. borealis, in Kyushu. In this study, the same isolate was paired with European and North American testers. The isolate was incompatible with $A$. borealis testers, but was compatible with $A$. mellea testers. Among the groups that were incompatible with all European and North American testers, we propose that Nag. E is a new species due to its unique basidiocarp morphology (21). Groups $\mathrm{G}$ and $\mathrm{H}$ represent $A$. singula and $A$. jezoensis, respectively, because the morphological characteristics of their basidiocarps are in accord with those described by Cha et al. (7).

All groups have a bifactorial heterothallism except for Nag. AM. Nag. AM is partially compatible with A. mellea of both Europe and North America. Some features of basidiocarp morphology (21), such as the absence of clamped basidia and presence of uninucleate basidia, are the same as in its European and North American counterparts. However, the monosporous colony morphology is crustose, as is the parental diploid culture. The nonheterothallic life cycle of Japanese A. mellea was studied with regard to the nuclear behavior during basidiospore formation and germination, and DNA content per nucleus at different developmental stages (24). Based on culture morphology, Cha and Igarashi

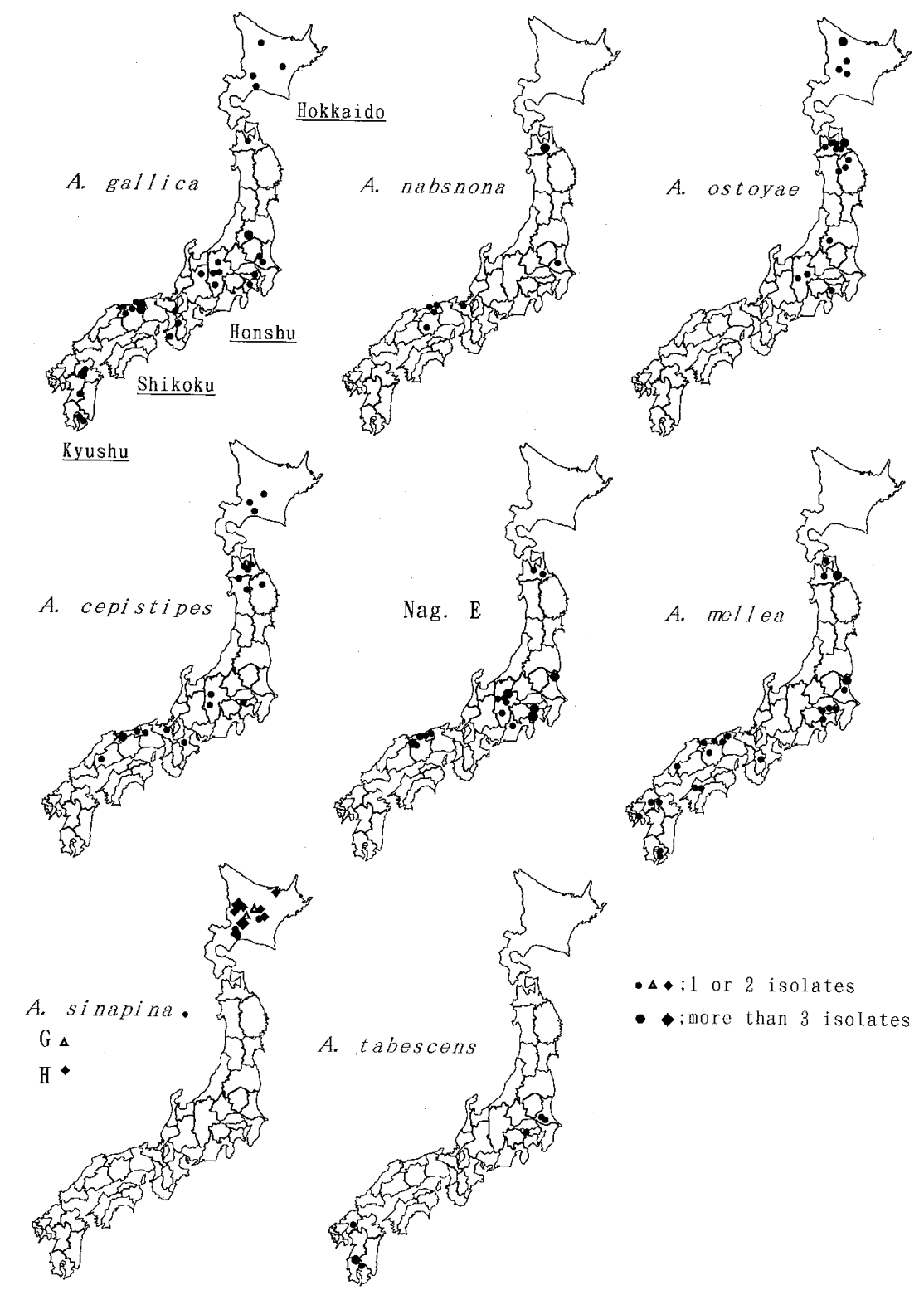

Fig. 2. Distribution of Armillaria spp. in Japan.

(6) supposed homothallism in A. mellea from Hokkaido and named it A. mellea subsp. nipponica. The similarities in monosporous culture colony morphology and the presence of clampless basidia suggest that they are a single group. In this paper, we refer to this group as "Japanese A. mellea."

A. gallica was the most abundant species collected. It was reported as a species common at low altitudes and at southern latitudes in the northern temperate hemisphere, and was considered a weak pathogen $(2,5,11,12)$. This also seems to be true in Japan (Fig. 2). In Japan, A. gallica is most commonly found on dead hardwoods and the soil surface.

A. nabsnona was reported as a species distributed on the west coast of North America (29). In this study, basidiocarps of A. nabsnona were collected at only 9 sites on Honshu island, central Japan (Fig. 2). The host range of A. nabsnona in North America is primarily hardwoods in riparian areas, particularly Alnus spp. (29). In Japan, basidiocarps were also collected from riparian areas.

A. ostoyae was reported as a species common in northern latitudes of the northern hemisphere $(2,5,11)$. In Japan, basidiocarps of this species were collected from Hokkaido and the northern part of Honshu, in the cool temperate zone (Fig. 2). This species is parasitic on Pinus densiflora (28) and Larix kaempferi in the northern region. From previous reports of Armillaria root rot occurring in northern conifer forests, almost all of the causal pathogens can be considered to be A. ostoyae, based on basidiocarp morphology (23).

A. cepistipes was reported as a species distributed with an inverse relationship between altitude and latitude (10). In Japan, it is distributed widely from north to south (Fig. 2).

Armillaria sinapina is considered to be a species with a relatively northern distribution in North America $(2,5,11)$. In Japan it is common in Hokkaido but not in the southern parts of Japan (Fig. 2).

A. mellea was reported to have a southern distribution in the northern hemisphere $(5,11)$. In this study, A. mellea was collected from both Honshu and Kyushu islands (Fig. 2). Cha and Igarashi (6) reported A. mellea subsp. nipponica from the southern part of Hokkaido, but it may be rare in Hokkaido. Especially in the southern part of Japan, this species seems to be a strong pathogen of $C$. obtusa.

A. tabescens was reported as a species common at low altitudes and at southern latitudes in the northern temperate hemisphere (14). In this study, basidiocarps of A. tabescens were collected from Kyushu islands and the southern part of Honshu.

A. singula and A. jezoensis may be restricted to Hokkaido. Nag. E was found only in Honshu and had no strong relationship to either host or substrate type. 
This study indicates that at least 10 biological species of Armillaria exist in Japan and perhaps explains some of the diverse parasitic activity noted in earlier studies of Armillaria root rot in Japan.

\section{ACKNOWLEDGMENTS}

We thank J. J. Guillaumin and J. B. Anderson for providing the Armillaria tester strains; T. Hattori for helping with the collection of basidiocarps; and G. Warwick for critical reading of the manuscript. This research was supported in part by a Grant-in-Aid for Scientific Research (No. 05454078) from the Ministry of Education, Science and Culture of Japan.

\section{LITERATURE CITED}

1. Anderson, J. B., and Ullrich, R. C. 1979. Biological species of Armillaria mellea in North America. Mycologia 71:402-414.

2. Banik, M. T., Paul, J. A., and Burdsall, J. J. Jr. 1995. Identification of Armillaria species from Wisconsin and adjacent areas. Mycologia 87:707-712.

3. Bérubé, J. A., and Dessureault, M. 1988. Morphological characterization of Armillaria ostoyae and Armillaria sinapina sp. nov. Can. J. Bot. 66:2027-2034.

4. Bérubé, J. A., and Dessureault, M. 1989. Morphological studies of the Armillaria mellea complex: Two new species, A. gemina and A. calvescens. Mycologia 81:216-225.

5. Blodgett, J. T., and Worrall, J. J. 1992. Distribution and hosts of Armillaria species in New York. Plant Dis. 76:166-170.

6. Cha, J. Y., and Igarashi, T. 1995. A note on Armillaria mellea subsp. nipponica subsp. nov. in Japan. Mycoscience 36:143-146.

7. Cha, J. Y., Sung, J. M., and Igarashi, T. 1992. Biological species and morphological characteristics of Armillaria mellea complex in Hokkaido: A. ostoyae and A. bulbosa. Res. Bull. Exp. For. Hokkaido Univ. 49:185-194.

8. Cha, J. Y., Sung, J. M., and Igarashi, T. 1994. Biological species and morphological characteristics of Armillaria mellea complex in
Hokkaido: A. sinapina and two new species, A. jezoensis and A. singula. Mycoscience 35:39-47.

9. Guillaumin, J. J., Anderson, J. B., and Korhonen, K. 1991. Life cycle, interfertility, and biological species. Pages 10-20 in: Armillaria root disease. C. G. Shaw and G. A. Kile, eds. U. S. Dep. Agric. For. Serv. Handb. 691.

10. Guillaumin, J. J., Mohammed, C., Anselmi, N., Courtecuisse, R., Gregory, S. C., Holdenrieder, O., Intini, M., Lung, B., Marxmuller, H., Morrison, D., Rishbeth, J., Termorshuizen, A. J., Tirro, A., and Van Dam, B. 1993. Geographical distribution and ecology of the Armillaria species in western Europe. Eur. J. For. Pathol. 23:321-341.

11. Guillaumin, J. J., Mohammed, C., and Berthelay, S. 1989. Armillaria species in the northern temperate hemisphere. Pages 27-43 in: Proc. 7th IUFRO Int. Conf. Root Butt Rots.

12. Harrington, T. C., and Rizzo, D. M. 1993. Identification of Armillaria species from New Hampshire. Mycologia 85:365-368.

13. Hood, I. A., Redfern, D. B., and Kile, G. A. 1991. Armillaria in plant hosts. Pages 122149 in: Armillaria Root Disease. C. G. Shaw, and G. A. Kile, eds. U. S. Dep. Agric. For. Serv. Handb. 691.

14. Kile, G. A., Guillaumin, J. J., Mohammed, C., and Watling, R. 1994. Biogeography and pathology of Armillaria. Pages 411-436 in: Proc. 8th IUFRO Int. Conf. Root Butt Rots.

15. Kile, G. A., and Watling, R. 1983. Armillaria species from south- eastern Australia. Trans. Br. Mycol. Soc. 81:129-140.

16. Kile, G. A., and Watling, R. 1988. Identification and occurrence of Australian Armillaria species including $A$. pallidula sp. nov. and comparative studies between them and nonAustralian tropical and Indian Armillaria. Trans. Br. Mycol. Soc. 91:305-315.

17. Korhonen, K. 1978. Interfertility and clonal size in the Armillaria mellea complex. Karstenia 18:31-42.

18. Matsushita, N., Fukuda, K., Nagasawa, E. Terashita, T., and Suzuki, K. 1996. Armillaria species in Japan identified by isozyme patterns with special reference to the biologica species of the Northern hemisphere. J. For. Res. 1:155-160.

19. Mohammed, C., Guillaumin, J. J., and Berthelay, S. 1994. Armillaria species identified in China and Japan. Mycol. Res. 98:607-613.

20. Muramoto, M. 1988. Occurrence of Armillaria root rot of Chamaecyparis obtusa. Forest Pest 37:65-70.

21. Nagasawa, E. 1991. Taxonomic reassessment of Armillaria mellea in Japan. Report for Grant-in-Aid for Scientific Research, No. 63560155, Tottori Mycol. Inst. Tottori, Japan.

22. Nomura, H. 1903. Root rot disease of mulberry trees in Kanagawa prefecture. Sanjihokoku 19:443-461.

23. Ono, K. 1965. Armillaria root rot in plantations of Hokkaido: Effect of topography and soil condition of its occurrence. (In Japanese with English summary.) Bull. Gov. For. Exp. Stn. 179:1-62.

24. Ota, Y., Fukuda, K., and Suzuki, K. The nonheterothallic life cycle of Japanese Armillaria mellea. Mycologia 90. In press.

25. Suzuki, K. 1996. Studies on biological species and ecological characteristics of Armillaria in Japan. Report for a Grant-in-Aid for Scientific Research, No. 05454078, Tokyo University, Japan.

26. Suzuki, K., Fukuda, K., Shiga, Y., Matsushita, N., and Terashita, T. 1994 Identification of Japanese biological species of Armillaria by isozyme patterns. Pages 376-382 in: Proc. 8th IUFRO Int. Conf. Root Butt Rots.

27. Terashita, T., and Chuman, S. 1989. Armillarias, isolated from the wild orchid, Galeola septentrionalis. Pages 364-370 in: Proc. 7th IUFRO Int. Conf. Root Butt Rots.

28. Terashita, T., and Sawaguchi, K. 1991. The pathogenicity of Armillaria root disease of Pinus densiflora in Aomori prefecture. Forest Pest 40:178-183.

29. Volk, T. J., Burdsall, H. H. Jr., and Banik, M. T. 1996. Armillaria nabsnona, a new species from Western North America. Mycologia 88:484-491. 